\title{
Glycymeris Longior Shells, an Alternative $\gamma$ Radiation Reference Detector
}

\author{
Carlos Fainstein \\ Centro Atómico Bariloche, CNEA and Instituto Balseiro, \\ UNC Av. E. Bustillo 9500, Bariloche, CP8400RN, Argentina
}

Received on 9 September, 2005; accepted on 31 October, 2005

\begin{abstract}
Gamma irradiation of biogenic calcium carbonate, from fossil shells of species Glycymeris Longior, results in the formation of $\mathrm{CO}^{2-}$ centers, as measured by electron paramagnetic resonance spectroscopy. The spectral line intensity increases linearly with increasing dose, without distortion, up to 50Gy. Fitting of the data yields the equation,
\end{abstract}

$$
\text { Dose }[\mathrm{Gy}]=-0.8(0.6)+0.0258(0.0004) \times[\mathrm{d} \chi \% / \mathrm{dH}] \mathrm{pp} / \mathrm{mg} \text {. }
$$

Over a year control measurements show no significative change in the data.

Keywords: Gamma irradiation; EPR

\section{INTRODUCTION}

The majority of invertebrate skeletal tissues are composed of the most stable crystalline polymorphs of $\mathrm{CaCO}_{3}$, calcite, and/or aragonite. A survey of the known biogenic minerals shows that approximately $80 \%$ are crystalline and $20 \%$ are amorphous ${ }^{(1)}$.

After gamma irradiation, fossil shells were reported to show electron paramagnetic resonance (EPR) spectral lines, that were attributed to different paramagnetic centers ${ }^{(2,3)}$, all related to the $\mathrm{CO}_{3}^{2-}$ molecule ion ${ }^{(4)}$.

For the $\mathrm{CO}^{2-}$ center, the accepted g-values are those given by S.A.Marshall et al. (3), with the z-axis along the C-O bond, the $\mathrm{x}$-axis in the [111] direction and the y-axis perpendicular to both. Those $\mathrm{g}$-values are $\mathrm{g}_{x}=2.00161, \mathrm{~g}_{y}=1.99727$, and $\mathrm{g}_{z}=2.00320$; with $\mathrm{g}=2.00232$ for the free electron value. Those data were obtained from optical grade single crystals, at $77 \mathrm{~K}$, when spectral lines are $30 \mathrm{mG}$ wide. Below $77 \mathrm{~K}$ the linewidths remain constant, but broaden for temperatures above $77 \mathrm{~K}$, and the spectral lines overlap. The total angular variation of the spectra is about $8 \mathrm{G}$.

In this work we report EPR data obtained from fossil sea shells of the family Glycymerididae, species Glycymerys Longior (GL), gamma irradiated by a ${ }^{137} \mathrm{Cs}_{55}\left(\mathrm{E}_{\gamma}=661 \mathrm{keV}\right)$ source. The irradiation rate was $0.24 \mathrm{~Gy} / \mathrm{min}$, and the estimated error in the values of Dose is less than $4 \%$.

We discuss the use of biogenic material obtained from GL fossil shells as a possible gamma irradiation detector and reference data storage, up to 50Gy Dose.

\section{SAMPLES}

A large number of fossil shells of GL were collected at the Atlantic sea shore, more precisely at Golfo San Matias, Argentina $\left(40^{\circ} 44^{\prime} \mathrm{S}, 64^{\circ} 57^{\prime} \mathrm{W}\right)$, selecting those that were whole and slightly polished by weather exposure. Three of those shells (about 25g total weight) were washed with a mild detergent, air dried and then pulverized with mortar and pestle. The powder thus obtained was passed through a $0.15 \mathrm{~mm} \times 0.15 \mathrm{~mm}$ sieve, and quantities of a few grams stored in polystyrene vials for further irradiation and storage. The EPR spectra of both the non-irradiated and irradiated samples, for each of the three shells, were found equal, and well within the spectrometer precision limit. Accordingly, we neglected EPR differences between the shells.

The XRD spectra of the material, see Fig. 1, show spectral lines that are assigned to the Aragonite and Calcite phases of Calcium Carbonate. Some XRD spectral lines remain unidentified, such as those located at $2 \theta=18.18^{\circ}, 34.19^{\circ}, 43.04^{\circ}$ and $50.32^{\circ}$.

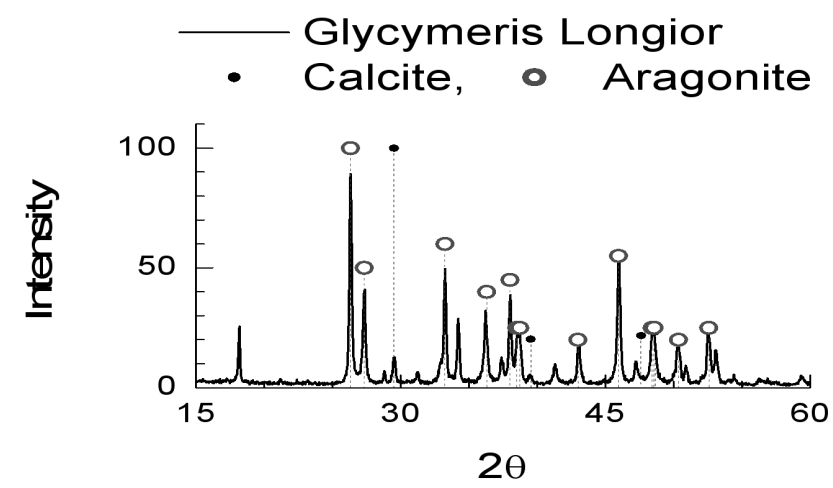

FIG. 1: XRD spectra of fossil shells, species Glycymeris Longior. Scattered points show data for Aragonite (open circles) and Calcite.

\section{EPR OF NON IRRADIATED SAMPLES}

The EPR spectra of the non irradiated sample are given in Fig. 2. The spectra suggest the presence of a radical interacting with two equivalent protons. The EPR parameters that fit the spectra of Fig. 2 are $g=2.0032$ (central line width $0.07 \mathrm{mT}$ ), with a hyperfine splitting of $0.9 \mathrm{mT}$, and spectral line widths of $0.10 \mathrm{mT}$, and $0.14 \mathrm{mT}$. The difference between the shape of these spectra and that of the irradiated samples is negligible. 


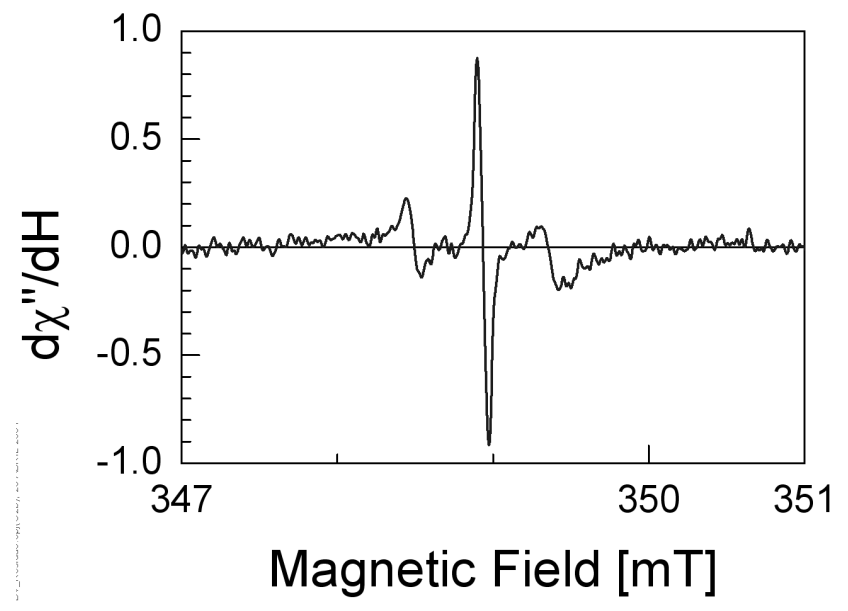

FIG. 2: EPR spectra of non-irradiated Glycymeris Longior fossil shells.

\section{EPR OF $\gamma$-IRRADIATED SAMPLES}

Figure 3 shows the EPR spectral lines of irradiated samples of GL shells, as recorded, for dose 0Gy, 4.8Gy and 48Gy. The spectral lines enclosed in circles are $\mathrm{Mn}^{2+}$ reference signals, from a single crystal of $\mathrm{MgO}$.

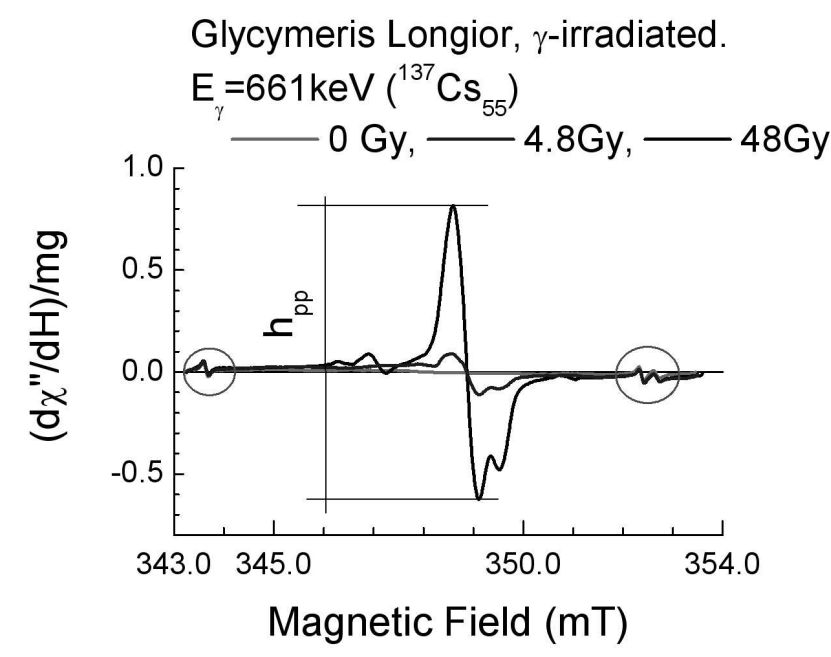

FIG. 3: EPR spectral lines of irradiated fossil shells of Glycymeris Longior.

Samples were irradiated in the dose range as required for blood irradiation protocols, in 5Gy steps, up to 50Gy. Known amounts of sample (about 100mg each) were lightly packed into $3 \mathrm{~mm}$ diameter pure quartz tubes, and then sealed. In order to compare quantitatively the EPR spectrum for the different irradiation doses, the following corrections were made,

1. Normalization of the spectra to a common value of spectrometer frequency,

2. Normalization to a common value for the spectrometer signal gain, using as a reference, the III and IV $\mathrm{Mn}^{2+} \mathrm{EPR}$ spectral lines from a $\mathrm{MgO}$ crystal, fixed to the sample holder,

\section{Glycymeris Longior, $\mathrm{E}_{\gamma}=661 \mathrm{keV}\left({ }^{137} \mathrm{Cs}_{55}\right)$}

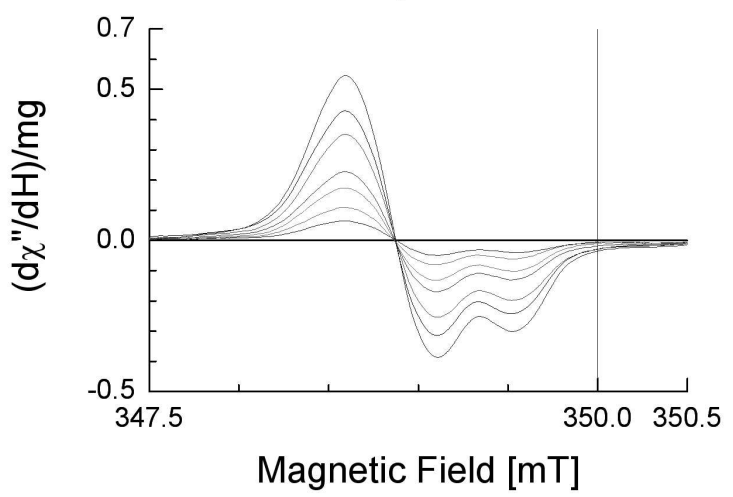

FIG. 4: a) Normalized EPR spectral lines from fossil shells of Glycymeris Longior, for Dose in the range $4.8 \mathrm{~Gy}$ to $48.0 \mathrm{~Gy}$.

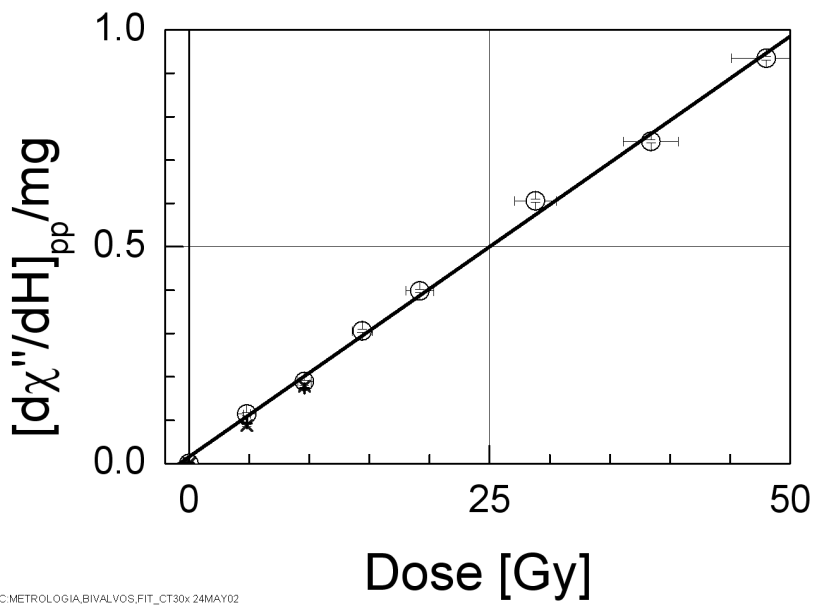

FIG.4: b) EPR peak-to-peak amplitude for Glycymeris Longior shells as a function of $\gamma$ irradiation dose. The EPR amplitude from unirradiated shells has been subtracted.

and

3. The EPR spectrum for the non irradiated sample was subtracted from all EPR spectra of the irradiated samples.

The final result after applying these corrections is shown in Fig. 4a, were it should be noted that the spectral line-shape remains unchanged, and that its amplitude, $[\mathrm{d} \chi " / \mathrm{dH}]_{p p} / \mathrm{mg}$, increases with increasing Dose. The spectral line-shape can be simulated with the parameters: $\mathrm{g}_{x}=2.0017, \mathrm{~g}_{y}=1.9975$ and $\mathrm{g}_{z}=2.0033$; [with line-widths $\Delta \mathrm{H}_{x}=0.325 \mathrm{mT}, \Delta \mathrm{H}_{y}=0.400 \mathrm{mT}$, and $\left.\Delta \mathrm{H}_{z}=0.375 \mathrm{mT}\right]$. These values agree with those given by Marshall et al. (3) for the $\mathrm{CO}_{2}^{-}$center, in an optical grade $\mathrm{CO}_{3} \mathrm{Ca}$ single crystal.

In Figure $4 \mathrm{~b}$ we show the peak-to-peak amplitude, as a function of $\gamma$-dose, up to $50 \mathrm{~Gy}$. The data points shown in Figure $4 \mathrm{~b}$ are obtained from EPR spectral lines of individual samples, measured at different times, over a one year period. The linear fitting of the data is given by the expression, $[\mathrm{d} \chi " / \mathrm{dH}]_{p p} / \mathrm{mg}=0.016(0.002)+0.0194(0.0001) \times$ Dose $[\mathrm{Gy}]$. 


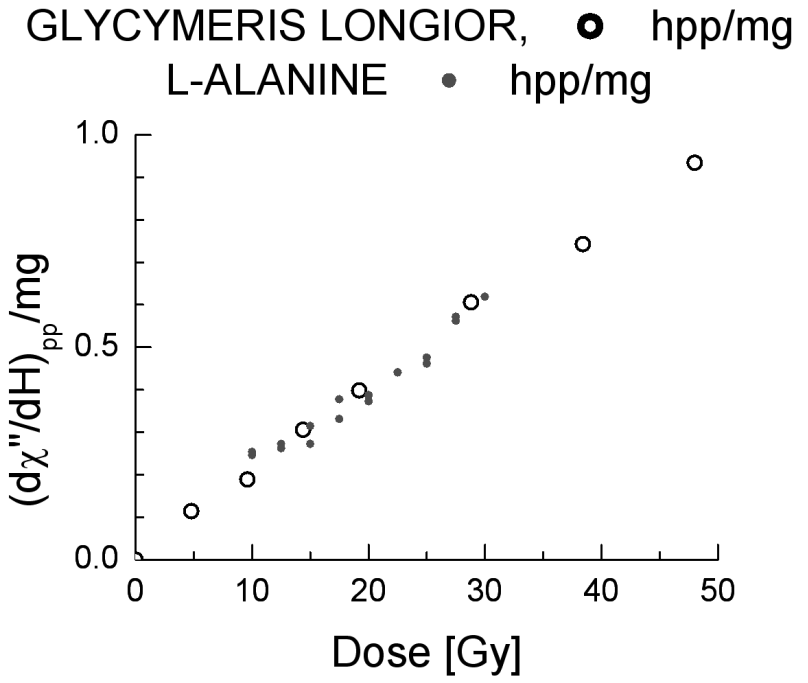

FIG. 5: EPR peak-to-peak amplitude as function of $\gamma$-dose for both Glycymeris Longior (open circles) and L-Alanine samples.

Finally, in Fig. 5 we compare data from both the amino acid L-Alanine ${ }^{(5)}$ and the biogenic mineral from GL, in the blood irradiation range, from $15 \mathrm{~Gy}$ to $50 \mathrm{~Gy}{ }^{(6,7)}$.

\section{CONCLUDING REMARKS}

Fossil shells from species Glycymeris Longior are extensively found at the atlantic shore, from Baia do Espírito Santo, Brasil $\left(20^{\circ} 17^{\prime} \mathrm{S}, 40^{\circ} 15^{\prime} \mathrm{W}\right)$ to Golfo San Matías, Argentina $\left(40^{\circ} 44^{\prime} \mathrm{S}, 64^{\circ} 57^{\prime} \mathrm{W}\right)$; the material of those shells is mostly polymorph calcium carbonate. A stable paramagnetic center is created on this biogenic mineral by $\gamma$-irradiation (up to 50Gy), which is identified by EPR spectroscopy as a $\mathrm{CO}^{2-}$ radical. The number of those radicals increases linearly with $\gamma$-dose, much at the same rate as it does for the aminoacid L-Alanine. For $\gamma$-dose larger than 50Gy the response $\left\{\left[(\mathrm{d} \chi \% / \mathrm{dH})_{p p} / \mathrm{mg}\right] /\right.$ Dose $\}$ is reduced, suggesting the onset of saturation

We conclude that the availability of this biogenic mineral, the stability of the radiation induced paramagnetic defect, and the response of the EPR line intensity versus $\gamma$-dose, favours the use of this solid as an EPR radiation detector in the blood irradiation range.
[1] H. A. Lowenstam and S. Weiner, On Biomineralization, Oxford University Press, New York, 1989.

[2] Ikeya, M. New applications of electron spin resonance: dating, dosimetry and microscopy. Singapore, World Scientific 1993. ISBN 9810211996.

[3] H. Peishua, L. Renyou, and J. Sizhao, P. Zicheng, and N. W. Rutter Appl. Radiat. Inst. 40, No. 10-12, 1119 (1989). (Int. J. Radiat. Appl Instrumen. Parr A)

[4] S. A. Marshall, A. R. Reinberg, R. A. Serway, and J. A. Hodges.
Mol. Phys. 8, 225 (1964).

[5] "Practice for use of the alanine-EPR dosimetry system". ISO/ASTM FDIS 51607:2001(E).

[6] "Practice for blood irradiation dosimetry". ISO/ASTM FDIS 51939:2001(E).

[7] C. Fainstein. E. Winkler, and M. Saraví, Applied Radiation and Isotopes 52, 1195 (2000) 